\title{
Le breton maritime à Plouhinec (Morbihan)
}

Maritime Breton in Plouhinec (56)

Serge Le Bozec

\section{CpenEdition}

\section{Journals}

Édition électronique

URL : http://journals.openedition.org//bl/354

DOl : $10.4000 / / b l .354$

ISSN : 2727-9383

\section{Éditeur}

Université de Bretagne Occidentale - UBO

\section{Édition imprimée}

Date de publication : 1 octobre 2018

Pagination : 155-175

ISBN : 979-10-92331-40-0

ISSN : 1270-2412

\section{Référence électronique}

Serge Le Bozec, "Le breton maritime à Plouhinec (Morbihan) », La Bretagne Linguistique [En ligne], 22 | 2018, mis en ligne le 01 mai 2020, consulté le 02 décembre 2020. URL : http:// journals.openedition.org//bl/354; DOl : https://doi.org/10.4000/lbl.354

\section{(c) (1)}

La Bretagne Linguistique est mise à disposition selon les termes de la Licence Creative Commons Attribution 4.0 International. 


\section{Serge LE BOZEC*}

\section{Le breton maritime à Plouhinec (Morbihan)}

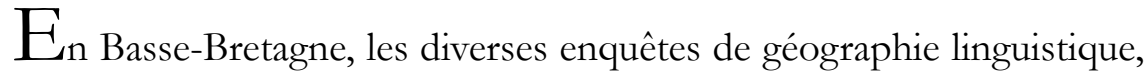
reportées dans des atlas, concernent plus l'intérieur que le littoral. Cela est tout simplement dû à une répartition des points d'enquête égale sur le territoire et la bande littorale n'en représente qu'une partie réduite. Mais ce qui est vrai pour les atlas, l'est moins pour d'autres ressources. Il existe beaucoup de monographies ou collectages sur des points de la côte : Sarzeau, l'Île-aux-Moines, Belle-Île, Plouharnel, Arradon, Riantec, Groix, Plogoff, Douarnenez, Lechiagat, l'île de Sein, Ouessant... par Ernault, Loth, Bernier, Le Diberder, Hammer, Le Besco, Audic, Ternes, Denez, Goyat, Sinou, Fagon, Dom Malgorn...

Le vocabulaire strictement maritime est globalement moins représenté. Il est donc intéressant de compléter les enquêtes par des termes spécifiques du bord de mer (ichthyonymes, phytonymes, environnement marin, vocabulaire technique de la pêche...) et de contribuer ainsi à une meilleure connaissance du vocabulaire de base et des éventuelles variations dialectales.

Cet article présente quelques résultats issus d'entretiens semidirigés en breton avec un informateur de la commune de Plouhinec, Morbihan. C'est un ancien pêcheur, actuellement en retraite à PortLouis. Il est né en 1932 à Kerzine en Plouhinec. Embarqué comme

\footnotetext{
* Enseignant de breton (s.le.bozec@orange.fr).
} 
mousse à l'âge de 14 ans sur les thoniers du port d'Étel, il a poursuivi sa carrière sur les chalutiers " pêche arrière » de Lorient et de Concarneau. Le breton est sa langue maternelle et il l'a pratiquée en compagnie des marins des thoniers, et a conservé de cette époque des termes bretons liés à cette activité professionnelle. Par conte, il ne l'a pas utilisée sur les chalutiers, ni en famille par la suite.

Les objectifs des entretiens avec l'informateur étaient les suivants :

- apporter des informations le plus exhaustives possible, ou du moins complémentaires, pour décrire le breton plouhinecois dans ses aspects phonologiques, morpho-syntaxiques, lexicaux...

- constituer un point d'enquête maritime supplémentaire au sein de l'ALCAM (Atlas Linguistique des Côtes de l'Atlantique et de la Manche). Cette partie du travail provient d'une suggestion de Daniel Le Bris, lui-même enquêteur dans le cadre de l'ALCAM, et le questionnaire a été intégralement soumis à l'informateur.

Les collectes de vocabulaire maritime étant peu nombreuses, voire lacunaires, il est indispensable d'interroger un maximum de locuteurs natifs afin de compléter les données.

Plouhinec est situé dans l'ancien canton de Port-Louis, dont les communes sont actuellement réparties entre la Communauté d'agglomération de Lorient et la Communauté de communes de Blavet Bellevue Océan. Cet ensemble est nettement délimité par la géographie physique, car il s'agit d'un territoire situé entre deux rias réduisant les communications vers l'ouest et l'est : la rade de Lorient et l'embouchure du Blavet à l'ouest, la « rivière d'Étel » de son nom local (ou ria d'Étel) à l'est. C'est l'océan Atlantique qui borde le territoire au sud. Du côté nord, il est difficile de mettre en évidence une frontière naturelle, mais la voie express RN 165 orientée est-ouest ferme cet espace et oriente les flux de personnes et marchandises en longeant les limites nord de Kervignac et Nostang. Cet axe routier accentue une littoralisation déjà ancienne du Sud-Bretagne. Le canton de Port-Louis est donc presque intégralement situé entre cet axe de circulation majeur et la mer, et peut être comparé à une véritable presqu'île. 
Noms administratifs des communes, leur graphie modernisée en breton et leur prononciation d'après l'informateur :

1 Gâvres

Gavr

ga:f

2 Kervignac

3 Locmiquélic

Logmikaelig

lomətfa'lə:t

4 Merlevenez

Brelewenez.

bərlaui'ni

5 Nostang

An Nostank, Lostank

ən nostãk

6 Plouhinec

Pleizhineg

ple:'ne:k

7 Port-Louis

Porah-Loeir.

8 Riantec

Rianteg

9 Sainte-Hélène

Santer Elenn

poRo 'lwajs

rijã'te:k

sãtezi'len

Entre Gâvres et les communes 6, 7 et 8 :

Petite Mer de Gâvres Mor Bihan Gavr à l'ouest :

La rade de Lorient Gwoaz̧ an Oriant / 'gweh ən o'rjãt

Lorient Gwoezh an Oriant

Lanester An Oriant ən o'rjãat

Groix

Lannarstêr

Groe

'mur bəjã 'ga:f

à l'est :

La rivière d'Étel Gwoaz̧ an Entell 'gweh ən in'tel Gwoezh an Entell

Le Bout du Havre Beg an Havr lan’'fte:r grwe

(embouchure de la rivière d'Etell, côté Plouhinec)

Étel

An Entell

ən in'tzl

Quiberon

Kiberen

tibə're:n

Belle-Île

(Enez) ar Gêrveur

ger'vø:r

La graphie « gwoazh » est un choix orthographique rendant compte de la prononciation vannetaise /we/ de l'ancienne diphtongue «oe» évoluée en /wa/ en breton KLT (Kernew - Leon - Treger) standard.

Linguistiquement parlant, la commune d'origine de l'informateur est située dans le pays vannetais bretonnant : la prononciation de l'ancien $/ \vartheta /$ a évolué en $/ \mathrm{h} /$ ou $/ \mathrm{x} /$, et non en $/ \mathrm{z} /$ comme généralement en KLT standard. Cette zone vannetisante correspond approximativement 
à la partie occidentale de l'ancien diocèse de Vannes. Elle regroupe traditionnellement deux grandes variantes dialectales qui apparaissent sur la carte 1 :

- le bas-vannetais, à l'ouest de la rade de Lorient

- le haut-vannetais, à l'est de la rade de Lorient.

La commune de Plouhinec est située en haut-vannetais.

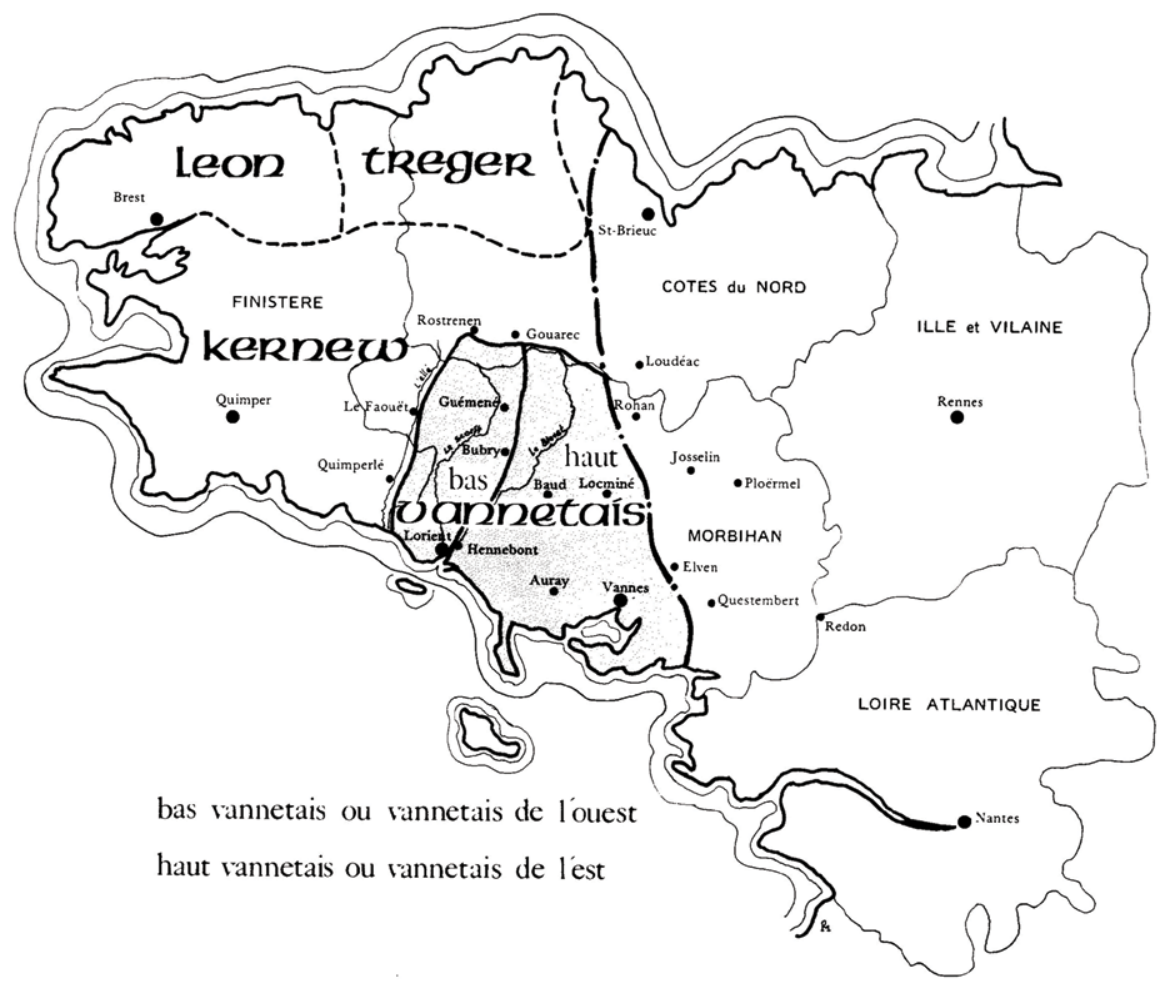

Carte 1 : Les grandes zones dialectales en Pays Vannetais bretonnant ${ }^{1}$.

Divers travaux de linguistique ont porté sur le breton de Plouhinec ou des environs :

- L'Atlas Linguistique de Basse-Bretagne (ALBB), de Pierre Le Roux,

1. Carte extraite de Meriadeg Herrieu, Le breton parlé, Vannes, Bleun Brug Bro Gwened, 1979.

2. Pierre Le Roux, Atlas linguistique de la Basse-Bretagne, Rennes, Plihon et Hommay, E. Champion, 1924. 
1924, où sont cartographiés 77 points d'enquête, dont le point 71 (Merlevenez) est le plus proche de Plouhinec (voir la carte 2 : les points d'enquête de l'ALBB).

- Le Nouvel Atlas Linguistique de Basse-Bretagne 3 (NALBB), de Jean le Dû, 2001 : 187 points d'enquête y sont regroupés, dont les points 170 (Nostang) et 171 (Plouhinec).

- Ichthyonymie bretonne ${ }^{4}$ d'Alain Le Berre, 1973. Enquêtes menées dans les années 1950. Le vocabulaire de la faune marine y est repertorié à partir d'enquêtes des années 1950 en 129 points littoraux de BasseBretagne. Au total, 430 espèces de poissons, de mollusques, de crustacés... y sont traitées. Les points les plus proches sont SaintCado à Belz (G11), Le Magoer à Plouhinec (G12) et Penn-Mane à Locmiquélic (G13).

- Les enquêtes ALCAM : Atlas Linguistique des Côtes de l'Atlantique et de la Manche. C'est un questionnaire conçu par Patrice Brasseur du département de linguistique de l'Université d'Avignon et qui porte sur le vocabulaire maritime. 109 points d'enquête sont répartis sur le littoral et couvrent plusieurs zones linguistiques, depuis le département du Nord (flamand) jusqu'à celui des Pyrénées Atlantiques (basque), en passant par les variétés d'oill, le breton, les variétés d'occitan (voir la carte 2).

Les questions, au nombre de 344, sont groupées par thèmes :

1 - Géomorphologie des côtes et de la mer (questions de 1 à 29)

2 - Les bateaux (de 31 à 56)

3 - Les pêcheurs (de 60 à 77)

4 - Aspect des poissons (de 80 à 96)

5 - Les poissons (de 101 à 185)

6 - Les coquillages (de 191 à 212)

7 - Zoophytes, annelidés, crustacés, céphalopodes (de 221 à 263)

8 - Les algues ( de 271 à 283)

9 - Les oiseaux marins (de 290 à 308)

10 - Techniques de pêche (de 311a à 344)

3. Jean LE Dû, Nouvel Atlas Linguistique de Basse-Bretagne, 2 vol., Brest, CRBC, 2001.

4. Alain LE BERRE, Ichthyonymie bretonne, 3 volumes, Brest, CRBC, 1973, il a été réédité en version numérique sous le titre Ichthyonymie bretonne, Atlas linguistique de la faune marine de Bretagne, Brest, CRBC et IUEM, 2008. 


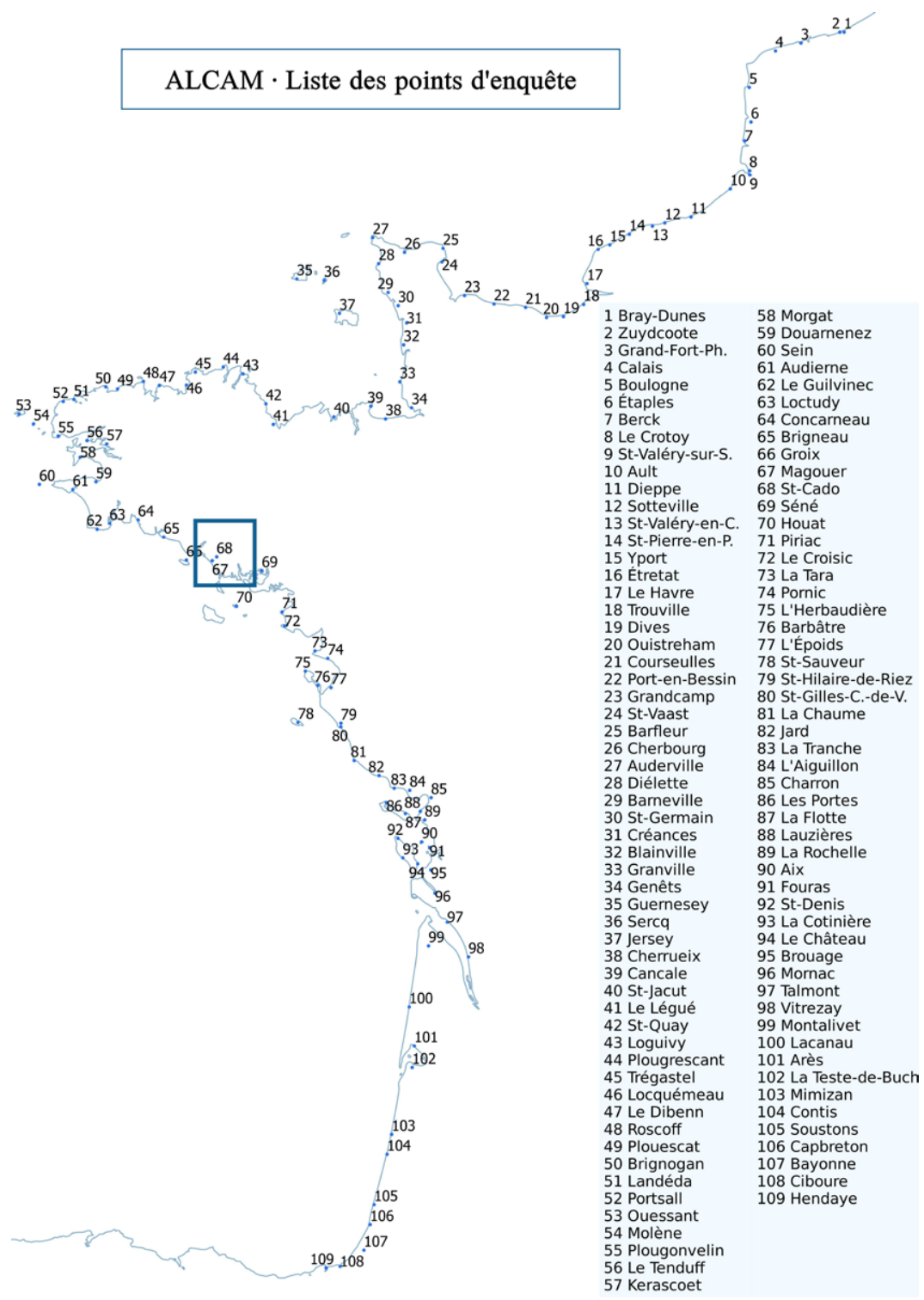

Carte 2 : Les points d'enquête de l'ALCAM (C) ALCAM, 2018). 
Le questionnaire de l'ALCAM a été soumis intégralement à l'informateur. Pour faciliter les entretiens, il a été fait usage du Guide de la faune et de la flore littorales des mers d'Europe, de Campbell et Nicholls, éditions Delachaux et Niestlé, ainsi que quelques cartes et diverses photographies.

Cet article ne présente qu'un échantillon des données issues des entretiens :

- l'accentuation et la phonologie

- quelques éléments de morphosyntaxe

- des exemples de termes maritimes recueillis dans le cadre de l'ALCAM

\section{L’accentuation et la phonologie}

\section{L'accentuation}

Comme en haut-vannetais de façon générale, la perception accentuelle tombe sur la dernière syllabe ${ }^{5}$.

- ur ga're:k

$\begin{array}{ll}\text { urgarreg } & \text { un rocher } \\ \text { Pleizhineg } & \text { Plouhinec } \\ \text { bihan } & \text { petit } \\ \text { jardrin } & \text { jardin } \\ \text { gouemon } & \text { des algues }\end{array}$

- ple:'ne:k

- bi'jã: / bə'jã:

- jar'drajn

gonemon

des algues

Parfois, la syllabe précédente (pénultième) peut être longue, surtout lorsqu'elle contient un [a].

- a:'yi:1

$\begin{array}{ll}\text { awel } & \text { vent } \\ \text { abred } & \text { tôt } \\ \text { arriv } & \text { arriver } \\ \text { abri } & \text { abri }\end{array}$

- a:'bərt

- a:'riu

abri

abri

\section{Le vocalisme}

Le haut-vannetais maritime est immédiatement identifiable par les locuteurs du vannetais par la prononciation spécifique des voyelles.

5. Sur ce phénomène, voir Patrick LE Besco, Trajedi Santez Julit ha Sant Sir he mab, Brest, Ar skol vrezoneg-Emgleo Breiz, 2001, p. 324 et Erwan LE PIPEC, Étude pluridimensionnelle d'un parler : description, émergence et aspects sociolinguistiques du breton de Malguénac, thèse de doctorat, Rennes, Université Rennes 2, 2008, p. 86-133. 
Ce fait est mentionné dans les anciennes grammaires ou méthodes du breton vannetais ${ }^{6}$. En voici des exemples :

- $[\mathrm{e}]>[\mathrm{i}]$

Le [e], voyelle fermée, devient encore plus fermé et est prononcé [i].

- mi 'ui:l ən 'trex

- in ty mã me a wel an traezh

je vois la côte

- a:'yi:1

en tu-mañ

de ce côté-ci

- də'rui

awel

du vent

- in am'zir ba'sat

darwe (₹), dalwe (₹)

en haut

- in'dru

en amzer based

autrefois

- i 'rawk

en-dro

de nouveau

- i'men i wez a:'riy a'zã

e-raog

avant

- di:n

e-menn eh ous arriv aze (n)

Où es-tu arrivé là?

- i'yi:1

den

ibuel / ubel

personne

haut

- i ma'gwe:r i wez a:'riy e Magoer eh ous arriv.

C'est au Magoer

que tu es arrivé.

- $[\mathrm{o}]>[\mathrm{u}]$

Le [o] long, surtout quand il est accentué, se ferme en [u]

- bə zu yr ga're:k

boud zo ur garreg

Il y a un rocher

- or mur

ar mor

la mer

- aui mune d'ən o'rjã:t

ewid moned d'an Oriant

pour aller à Lorient

- $[\mathrm{i}]>[\varepsilon j]$

La voyelle [i] longue accentuée devient [cj]

$-n \varepsilon j$

- on tej 'ncj

- yr '(h) $\varepsilon j$

- brə'lkj

- kər'lej ni

bon ti-ni

ur c'bi

brizbilli

Korneli (Sant) nous

notre maison (à nous)

un chien

des maquereaux

Saint-Cornély

6. Joseph LE BAYON, Grammaire bretonne du dialecte de Vannes, Vannes, Lafolye, 1896 ; A. GuILLEVIC et P. LE GofF, Grammaire bretonne du dialecte de Vannes, Vannes, Lafolye et de Malarzelle, 1931 ; Meriadeg Herrieù, Le breton parlé, Vannes, Bleun Brug Bro Gwened, 1979. 
- Certains « a » se prononcent [a:]

Le a long accentué devant consonne sonore est prononcé avec un point d'articulation postérieur.

$\begin{array}{lll}\text { - ur va:k } & \text { urvag } & \text { un bateau } \\ \text { - dərja:s } & \text { dariaz, } & \text { en bas } \\ \text { - ga:f } & \text { Gavr } & \text { Gâvres } \\ \text { - pla:ch } & \text { "plaj» } & \text { plage } \\ \text { - sã fja:k } & \text { Sant Fiag(r) } & \text { Saint-Fiacr }\end{array}$

- $[\varepsilon j]>[a j]$

Le groupe /عj/ majoritaire s'ouvre localement en /aj/.

- bə zu majn gətẽ

boud ro mein gete $(n t) \quad$ Ils ont des pierres

- poR lwajs

Porsh-Loeiz.

Port-Louis

- brajn

brein

pourri

- La prononciation du $[\varepsilon]$

Le haut-vannetais maritime est aussi caractérisé par une diphtongaison en [ja] ou [je] de la voyelle $\varepsilon$ accentuée. Mais cette modification n'est pas attestée chez l'informateur, sauf dans un mot:

- pjem pemp cinq

Le $\varepsilon$ est maintenu dans :

- frey (et non frjay) frew en forme, sain

- pen (et non pjan ni pjen) penn tête

\section{Le consonantisme}

Le consonantisme obéit à des règles précises dans l'ensemble du domaine bretonnant, et n'a pas d'alternative correcte ${ }^{7}$. La neutralisation des consonnes en finale absolue au profit de la dévoisée et la neutralisation en liaison devant voyelle au profit de la voisée est particulièrement nette à Plouhinec, y compris pour les consonnes appariées $\mathrm{s} / \mathrm{z}$ qui peuvent parfois faire exception par ailleurs.

7. Francis FAVEREAU, Grammaire du breton contemporain, Morlaix, Skol Vreizh, 1997. 


\begin{tabular}{|c|c|}
\hline En liaison devant voyelle & En finale absolue \\
\hline $\begin{array}{l}\text { kaz a 'dray gə’nət } \\
\text { kas « } a » \text { draoù genit } \\
\text { apporte tes affaires }\end{array}$ & $\begin{array}{l}\text { lãn a 'dray də gas } \\
\text { leun a draoù da gas } \\
\text { plein de choses à apporter }\end{array}$ \\
\hline $\begin{array}{l}\text { ur ga're:g azã } \\
\text { ur garreg aze( } \tilde{n}) \\
\text { un rocher là }\end{array}$ & $\begin{array}{l}\text { bo zu ur ga're:k } \\
\text { boud zo ur garreg } \\
\text { il y a un rocher }\end{array}$ \\
\hline $\begin{array}{l}\text { tud də’rja:z a'mã } \\
\text { toud dariaz / daziaz } \\
\text { tout en bas, là }\end{array}$ & $\begin{array}{l}\text { də’rja:s } \\
\text { dariaz / daziaz. } \\
\text { en bas }\end{array}$ \\
\hline $\begin{array}{l}\text { i we:z i por 'lwajs } \\
\text { eh ous e Porzh-Loeiz } \\
\text { tu es à Port-Louis }\end{array}$ & $\begin{array}{l}\mathrm{i} \text { 'men i 'we:s } \\
\text { e-menn eh ous? } \\
\text { Où es-tu? }\end{array}$ \\
\hline $\begin{array}{l}\text { ti 'baz in nay 'dy } \\
\text { te a bas en naou du } \\
\text { Tu passes des deux côtés }\end{array}$ & $\begin{array}{l}\text { ti 'bas } \\
\text { te a bas } \\
\text { Tu passes }\end{array}$ \\
\hline 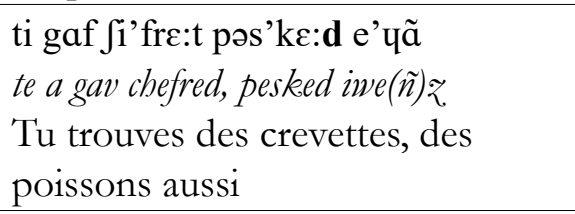 & $\begin{array}{l}{[\ldots] \text { poskə 'pla:t : }} \\
{[\ldots] \text { pesked plad }} \\
\text { des poissons plats }\end{array}$ \\
\hline $\begin{array}{l}\text { ti 'lag ə: } \\
\text { Te a lak, eu... } \\
\text { Tu mets ... euh }\end{array}$ & \\
\hline $\begin{array}{l}\text { i’mãd i fa:'rajn gwe'mõ } \\
\text { Emant [an dud] é serriñ goemon. } \\
\text { Ils ramassent du goémon }\end{array}$ & \\
\hline $\begin{array}{l}\text { or bâ'gau bijãn ə 'baz in ty 'sy } \\
\text { Ar bagoù bihan a bas en tu «su » } \\
\text { Les petits bateaux passent du côté } \\
\text { sud }\end{array}$ & $\begin{array}{l}\text { Mare'jay bi'jã } \\
\text { mareioù bihan } \\
\text { de petites marées }\end{array}$ \\
\hline
\end{tabular}

\section{La particule déictique «se(n) »}

La particule déictique «se(n) » s'inscrit dans un paradigme d'allophones en fonction de son environnement phonologique. Il en existe trois variantes utilisées comme indiqué dans le tableau ci-après. 


\begin{tabular}{|c|c|c|}
\hline $\begin{array}{c}1: \text { «se }(\mathrm{n}) » \\
\text { en position interne } \\
\text { devant consonne }\end{array}$ & $\begin{array}{c}2: \text { «se }(\mathrm{n}) » \\
\text { en position interne } \\
\text { devant voyelle }\end{array}$ & $\begin{array}{c}3: \text { : } \operatorname{se}(\mathrm{n}) » \\
\text { en finale absolue }\end{array}$ \\
\hline $\begin{array}{c}\text { azə zu 'tyt } \\
\text { aze zo tud } \\
\text { là il y a du monde } \\
\text { azə zu rə’sak } \\
\text { aze zo resak } \\
\text { là il y a du ressac } \\
\text { in ty sə marsã } \\
\text { en tu-se marse(n) } \\
\text { de ce côté-là peut-être }\end{array}$ & $\begin{array}{c}\text { i mosən ‘ } \tilde{a} \\
e-m o d-s e(n) \text { eo-eñv } \\
\text { il est comme ça }\end{array}$ & $\begin{array}{c}\text { ur gareg a'zã } \\
\text { ur garreg azee(n) } \\
\text { un rocher, là } \\
\text { dy'zã } \\
\text { du-se(n) } \\
\text { là } \\
\text { in ty sã } \\
\text { en tu-sen } \\
\text { de ce côté-là } \\
\text { in ty sə mar'sã } \\
\text { en tu-se marse(n) } \\
\text { de ce côté-là peut-être } \\
\text { gu'di nə’sã } \\
\text { goude neuse(n) } \\
\text { après donc } \\
\text { aanə'sã } \\
\text { abanese(n) } \\
\text { de là }\end{array}$ \\
\hline
\end{tabular}

Le tableau indique que :

- l'allophone sə / zə est utilisé en position interne devant consonne, la voyelle de se(n) est réduite à la voyelle centrale moyenne.

- l'allophone sən / zon est utilisé en position interne devant voyelle, la consonne « $\mathrm{n}$ » est insérée dans une liaison intervocalique.

- l'allophone sã / zã est utilisé en finale absolue, la voyelle est ouverte et nasalisée et le $\mathrm{n}$ final est amui.

Tout indique que l'étymon de la particule déictique est de forme «sen », et que la forme courante «se » en est une variante par réduction. 
On remarque de plus que le " $\mathrm{s}$ » initial de « se(n) » est sonorisé dans les adverbes de lieu aze at du-se (duze). Il demeure sourd dans les autres adverbes : «e mod-se(n), neuse(n), ahanese(n), marse(n)». Enfin, employé comme élément final du déterminant démonstratif « ər ... $\mathrm{sə}(\mathrm{n}) /$ ən ... - sə(n) », il n’est pas non plus sonorisé.

\section{Quelques éléments de morphosyntaxe}

Les tableaux ci-dessous ne regroupent que les pronoms personnels relevés au cours des entretiens avec l'informateur.

\begin{tabular}{|c|c|c|c|c|c|c|}
\hline & \multicolumn{3}{|c|}{$\begin{array}{c}\text { Pronoms personnels indépendants } \\
\text { et sujets }\end{array}$} & \multicolumn{3}{|c|}{$\begin{array}{l}\text { Pronoms personnels } \\
\text { COD antéposés }\end{array}$} \\
\hline & $\begin{array}{l}\text { Transcription } \\
\text { phonologique }\end{array}$ & $\begin{array}{l}\text { Graphie } \\
\text { courante }\end{array}$ & Traduction & $\begin{array}{l}\text { Transcription } \\
\text { phonologique }\end{array}$ & $\begin{array}{l}\text { Graphie } \\
\text { courante }\end{array}$ & Traduction \\
\hline $1 \mathrm{~S}$ & $\mathrm{mi} / \mathrm{m} ə$ & me & $\mathrm{moi} / \mathrm{je}$ & & & \\
\hline $2 \mathrm{~S}$ & ti & te & toi/tu & & & \\
\hline $3 \mathrm{~S} \mathrm{~m}$ & jõ / jã & eñv & lui/il & $\tilde{\mathrm{a}}$ & hañ / hoñ & le \\
\hline $3 \mathrm{~S} \mathrm{f}$ & $\varepsilon j$ & hi & elle & & & \\
\hline $1 \mathrm{P}$ & ncj & $\mathrm{ni}$ & nous & & & \\
\hline $2 \mathrm{P}$ & પદj & c'hwi & vous & & & \\
\hline $3 \mathrm{P}$ & in & int & eux/ils/elles & $\mathrm{u} / \varepsilon$ & o / hê & les \\
\hline
\end{tabular}

\begin{tabular}{|l|c|c|c|c|c|c|}
\hline \multirow{3}{*}{} & \multicolumn{3}{|c|}{$\begin{array}{c}\text { Pronoms personnels } \\
\text { prépositionnels (avec da) }\end{array}$} & \multicolumn{3}{c|}{$\begin{array}{c}\text { Pronoms personnels } \\
\text { prépositionnels (avec get) }\end{array}$} \\
\cline { 2 - 7 } & $\begin{array}{l}\text { Transcription } \\
\text { phonologique }\end{array}$ & $\begin{array}{c}\text { Graphie } \\
\text { courante }\end{array}$ & Traduction & $\begin{array}{c}\text { Transcription } \\
\text { phonologique }\end{array}$ & $\begin{array}{c}\text { Graphie } \\
\text { courante }\end{array}$ & Traduction \\
\hline $1 \mathrm{~S}$ & din-mi & din-me & me / à moi & & & \\
\hline $2 \mathrm{~S}$ & dət & dit & te / à toi & gənət & genit & avec toi \\
\hline $3 \mathrm{P}$ & & & & $\begin{array}{l}\text { gəte } \\
\text { gətẽ }\end{array}$ & $\begin{array}{c}\text { gete } \\
\text { getent }\end{array}$ & $\begin{array}{c}\text { avec } \\
\text { eux } / \text { elles }\end{array}$ \\
\hline
\end{tabular}

En voici quelques exemples :

- Le pronom indépendant $1 \mathrm{~S}$ est réduit à l'allophone atone /mə/ : il n'y a pas d'insistance particulière sur le sujet du verbe.

mə sa'uع gwe'mõ / Me a save goemon / Je montais du goémon. 
- Les pronoms 3P COD antéposés u/E (o/hê) s'emploient comme variantes libres.

ncj u lake a'r gart $\varepsilon 1$ ha nej u gas $\varepsilon$ dərui

Ni o lake àr ar garrikell ba ni o " gase » darwez:

Nous les mettions sur la brouette et nous les apportions en haut.

nєj $\varepsilon$ sau $\varepsilon$ dərui

Ni hê save darwez.... nej $\varepsilon$ lake in tawen

ni hê lake en tewenn.

Nous les montions en haut : nous les mettions sur la dune

- Le pronom S3m postposé à l'auxiliaire avoir dans le verbe composé est audible sous sa variante / $\tilde{a} /$ au cours des entretiens.

ma rə lə’kəl ər 'me:n عl masã tro'vət

Ema red leuskel ar mên evel ma 'c'b eus-hañ «troved».

Il faut laisser la pierre comme tu l'as trouvée.

$\mathrm{NB}$ : La liaison entre « ac'b eus» et ce pronom 3Sm est sourde en raison du h initial d'origine du pronom : /masã/.

- Les pronoms COD antéposés / $\mathrm{u} /$ transcrit « O » de la $3^{\mathrm{e}}$ personne du pluriel s'emploient à la forme affirmative ou négative :
tu have
Te o c'have.
Tu les trouvais.
nu have Jat N'o c'bavez ked
Tu ne les trouvais pas.

Mais quelques occurrences de la forme $3 \mathrm{P}$ « ane乏he $(n t) »$ apparaissent dans des indépendantes négatives. Il y a deux interprétations possibles :

1) on a affaire ici au pronom prépositionnel anezhe, substitut du COD :
uile $\int \partial \operatorname{nĩn}(\mathrm{t}) / \operatorname{nēn}(\mathrm{t})$

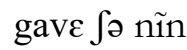
Ne welez ked (a)nehe (nt).
Ne gaver. ked (a)nehe (nt).

Tu ne les vois pas.

Tu ne les trouves pas.

Cet emploi n'est normalement pas attesté en haut-vannetais, où on dit «N'o gwelez ked». Par contre, cette structure syntaxique est connue en bas-vannetais, en alternance avec le système du haut-vannetais. A-t-on ici une expansion vers l'est d'un usage bas-vannetais provenant du pays de Lorient? 
2) ou bien, il s'agit d'un pronom 3P partitif :

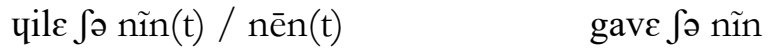

Ne welez ked (a)nehe $(n t)$. Ne gaver. ked (a)nehe $(n t)$.

Tu n'en vois pas (des poissons). Tu n'en trouves pas (des poissons).

L'emploi de la préposition a après un verbe négatif pour rendre un sens partitif est par contre courant en breton, y compris en hautvannetais. C'est cette interprétation qui est la plus plausible.

- Il ne faut pas confondre l'emploi décrit dans le paragraphe précédent avec celui du pronom prépositionnel 3P «anezhe(nt)», employé systématiquement, voire obligatoirement, dans une proposition à la forme négative afin de préciser le genre et le nombre du sujet de $3^{\mathrm{e}}$ personne. L'accord verbal en nombre n'est pas réalisé : le verbe reste à la $3^{\mathrm{e}}$ personne du singulier même si le sujet est pluriel.

ve t $\int a$ debə 'nĩn

Ne vez, ked debred (a)nehe (nt).

Littéralement : N'est pas mangé quant à eux.

Ils ne sont pas mangés. $=$ On ne les mange pas.

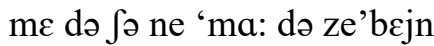

Mes, n'eo ked (a)nezhe mad da zebriñ.

Littéralement : Mais, n'est pas quant à eux bon à manger.

Mais, ils ne sont pas bons à manger.

wer t $\int \partial$ 'nõ

Ne oar ked anezhoñ.

Littéralement: Ne sait pas quant à lui.

Il ne sait pas.

da tfə 'ncj

Nend a ked anezhi.

Littéralement : Ne va pas quant à elle.

Elle ne va pas. 


\section{Termes maritimes de I'ALCAM}

L'autre objectif fondamental des entretiens avec l'informateur était de collecter les formes locales (en breton, voire en français local) d'une liste de termes maritimes proposés dans le questionnaire de l'ALCAM.

Dans les tableaux ci-dessous sont répertoriés les lexèmes ayant trait au thème 1 (Géomorphologie des côtes et de la mer, questions de 1 à 29) et au thème 5 (Les poissons, questions de 101 à 185).

Thème 1 : Géomorphologie des côtes et de la mer

\begin{tabular}{|c|c|c|c|}
\hline Français & API & Breton & Exemples \\
\hline un rocher & yr ga're:k & ur garreg & $\begin{array}{l}\text { bə zu yr ga're:k } \\
\text { Boud zo ur garreg. } \\
\text { Il y a un rocher. }\end{array}$ \\
\hline la dune & ən ta'yદn & an tewenn & $\begin{array}{l}\text { bo zu 'jewd in ta' yen } \\
\text { Boud ro yeot en tewenn. } \\
\text { Il y a de l'herbe sur la dune. }\end{array}$ \\
\hline du sable & sẽ $: p$ & señbl & \\
\hline la côte & ən 'tre: $\mathrm{x}$ & an traeß̧ & $\begin{array}{l}\text { auə mu'ne də da'pwi mi'skle:t } \\
\text { ve lâ'rət jam dən 'tre:x } \\
\text { Ewid moned da daponed meskled... } \\
\text { e vez lâred eh amp d'an traezh. } \\
\text { Pour aller chercher des moules.. } \\
\text { on dit que nous allons à la côte }\end{array}$ \\
\hline la mer & ər 'mu:r & ar mor & $\begin{array}{l}\text { ma ər 'mur i ti' Jen mar'sã } \\
\text { Ema ar mor é tiskenn marsen. } \\
\text { La mer descend peut-être. }\end{array}$ \\
\hline l'océan & $\begin{array}{l}\text { ər mu:r } \\
\text { 'bra:s }\end{array}$ & ar mor bras & $\begin{array}{l}\text { ma jez ir mur 'bra:s } \\
\text { Ma yez er mor braz... } \\
\text { Si tu vas sur l'océan... }\end{array}$ \\
\hline en haut & də'rui & $\begin{array}{l}\text { dalwez }= \\
\text { darwez. }\end{array}$ & $\begin{array}{l}\text { pə də or 'mur də'rui } \\
\text { pad eo ar mor darwez } \\
\text { Quand la mer est haute }\end{array}$ \\
\hline en bas & də’rja:s & $\begin{array}{l}\text { daziaz }= \\
\text { dariaz }\end{array}$ & $\begin{array}{l}\text { ən 'dø:r a 'fom də’rja:s } \\
\text { An doùr a chom daria: } \\
\text { La mer reste basse. }\end{array}$ \\
\hline
\end{tabular}




\begin{tabular}{|c|c|c|c|}
\hline marée & ma:'re & mare & $\begin{array}{l}\text { ər 'mur ə za bə'dag ən no'stãk pəd ə } \\
\text { ma:re 'bra:s } \\
\text { Ar mor a za bedag an Nostank pad eo } \\
\text { mare braz: } \\
\text { La mer vient jusqu'à Nostang quand } \\
\text { il y a de grandes marées. }\end{array}$ \\
\hline marées & mare'jay & mareioù & $\begin{array}{l}\text { Mare'jay bi'jã } \\
\text { mareioù bihan } \\
\text { de petites marées }\end{array}$ \\
\hline $\begin{array}{l}\text { flot: marée } \\
\text { montante }\end{array}$ & & & $\begin{array}{l}\text { ma or 'mur i tu'ne də’rui } \\
\text { Ema ar mor é toned darwez. } \\
\text { La mer vient en haut }\end{array}$ \\
\hline $\begin{array}{l}\text { jusant : } \\
\text { marée } \\
\text { descendante }\end{array}$ & & & $\begin{array}{l}\text { ma ər 'mur i ti' fen mar'sã } \\
\text { Ema ar mor é tiskenn marsen. } \\
\text { La mer descend peut-être. } \\
\text { on 'dø:r ə zifal 'mat } \\
\text { An doùr a zichal mat. } \\
\text { La mer descend bien. }\end{array}$ \\
\hline Mi-marée? & in i zark & $\begin{array}{l}\text { en e jarg/ } \\
\text { jark? }\end{array}$ & $\begin{array}{l}\text { pə za ən 'dø:r də’rui... } \\
\text { ən 'dø:r ə a:'riu in i zark... } \\
\text { gudi nə’sã... } \\
\text { pə də iuદ'loh ər 'mur } \\
\text { azə ve la:rə nə’sã ... } \\
\text { ima ər 'mur də’rui } \\
\text { Pa za an doùr darwę... } \\
\text { an doùr a arriv en e jarg / jark ?... } \\
\text { goude neusen ... } \\
\text { pad eo uhelloc'b ar mor, } \\
\text { aze (e) ve lâred neusen : } \\
\text { « Ema ar mor darwez. » } \\
\text { Quand la mer monte ... } \\
\text { l'eau arrive à mi-marée? } \\
\text { ensuite alors ... } \\
\text { quand la mer est plus haute, } \\
\text { là, on dit alors : } \\
\text { « La mer est en haut. » }\end{array}$ \\
\hline
\end{tabular}




\begin{tabular}{|l|l|l|}
\hline basse mer, & & - pəd ər 'mu:r də’rja:s \\
pleine mer & Pad eo ar mor dariaz \\
& Quand la mer est basse. \\
& - pəd ə ər 'mu:r də'ryi \\
& Pad eo ar mor darwez \\
& Quand la mer est haute. \\
& - pəd ə i' yi:l ər 'mu:r \\
& Pad eo uhel ar mor \\
& Quand la mer est haute. \\
\hline
\end{tabular}

\section{Commentaires :}

1) Le terme tre:x / traę̧ ne désigne pas le sable, mais la côte, le

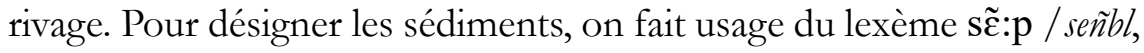
comparable au français sable.

2) Le terme awt / aod est aussi utilisé.

3) Quant au lexème homophone tre: $\mathrm{x} /$ treizh , il est utilisé dans le toponyme plouhinecois ən trex 'kux / « an Treizh Kozh», connu sous son nom français «le Vieux Passage ».

4) Une expression inconnue pour nous - et des dictionnaires jusqu'à son utilisation spontanée par l'informateur lors de la partie de l'entretien concernant le vocabulaire des marées a fait l'objet d'une explication de sa part en breton :

pə za ən 'dø:r də'rui ... ən 'dø:r ə a:'riu in i zark... gudi nə’sã ... pə də iц $\varepsilon$ 'loh ər 'mur azə ve la:rə nə'sã ... ima ər 'mur də'rui

$\mathrm{Pa}$ za an doùr darwez... an doùr a arriv en e jarg / jark ?... Goude neusen ... pad eo uhelloc'h ar mor, až (e) ve lâred neusen : "Ema ar mor darwez: 》 Quand la mer monte ... l'eau arrive dans son/sa jark/jarg... ensuite alors ... quand la mer est plus haute, là, on dit alors : « La mer est en haut. »

Appliquée à la marée montante, « en e jark /jarg » semble signifier que la mer a atteint le niveau de la mi-marée et qu'elle couvre déjà bien l'estran. 
Thème 5 : Les poissons

\begin{tabular}{|c|c|c|c|}
\hline Français & API & Breton & Nom scientifique \\
\hline poissons & pəske:t & pesked & Pisces \\
\hline moules & mis'kle:t & meskled & Mytilus edulis \\
\hline crabes & krãkə’ḑe:t & krankeged & Brachyura \\
\hline plies & plajn'se:t & pleised & Pleuronectes platessa \\
\hline araignées de mer & kaminiriõ & kemenerion & Maia squinado \\
\hline dormeurs, tourteaux & kouskəriõ & kouskerion & Cancer pagurus \\
\hline palourdes & palurd $\varepsilon: t$ & palourded & Venerupis decussata \\
\hline maquereaux & brəlcj & briz̧illi & Scomber scombrus \\
\hline $\begin{array}{l}\text { gourlazeaux } \\
\text { crénilabres, non } \\
\text { distingués des vieilles. }\end{array}$ & $\begin{array}{l}\text { gurlazãne:t } \\
\text { gurlaze:t }\end{array}$ & $\begin{array}{l}\text { gourlazaned } \\
\text { gourlazed }\end{array}$ & Crenilabrus sp. \\
\hline
\end{tabular}

Les termes plouhinecois peuvent être comparés à leurs équivalents des communes proches ou plus lointaines, repertoriés lors d'enquêtes similaires, et ce de façon d'autant plus pertinente que le système phonologique particulier du haut-vannetais maritime aura été précisément caractérisé par rapport au vannetais et aux autres dialectes bretons.

À titre d'exemple, le lexème /brolcj/ briz̧billi utilisé par l'informateur est bien à interpréter comme le nom «brizhilli » comportant le suffixe «i » du pluriel, et non comme un hypothétique «breuleilh» d'interprétation délicate. La forme / brəlej/ correspond parfaitement aux évolutions phonétiques et phonologiques locales réflexes de prononciation de Plouhinec : le phonème $/ \mathrm{h} /$ intervocalique y est muet; le /i/ inaccentué perd sa couleur vocalique et tend vers un schwa /ə/; le /i/ final accentué s'allonge et se diphtongue en / $\mathrm{cj} /$. Cette forme / bralcj/ s'inscrit donc dans la continuité de variantes comme /brehi'li/, /'bri:li/ ou /bre'zili/, cités dans Ichtyonymie bretonne ${ }^{8}$.

8. Alain LE BERRE, Ichthyonymie bretonne, 3 volumes, Brest, CRBC, 1973. 


\section{Conclusion}

Le breton de Plouhinec n'a pas encore fait l'objet d'étude précise, à l'instar de celui des variétés locales des alentours : Plouharnel $^{9}$, Groix $^{10}$, pays de Lorient ${ }^{11}$ et Languidic ${ }^{12}$. Il subsiste encore de - rares - locuteurs natifs dans cette commune, ainsi que dans les autres communes de l'ex-canton de Port-Louis. Une étude d'ensemble du breton de cette presqu'île située entre la rade de Lorient et la rivière d'Étel permettrait de combler le hiatus dialectologique le long de cette portion du littoral morbihannais et de contribuer à la compréhension des continuités et ruptures du breton local.

D'autre part, le questionnaire de l'ALCAM peut servir de guide pour mener les enquêtes linguistiques. Cela permettrait de collecter de surcroît des termes maritimes, afin d'enrichir le thésaurus déjà répertorié dans les fichiers de l'ALCAM. En combinant ces données à celles de Ichthyonymie bretonne, de l'Atlas linguistique de la faune marine de Bretagne, de l'Atlas Linguarum Europae (ALE), de l'Atlas Linguistique Roman (AliR), et toutes autres sources présentes dans les dictionnaires de langues, il est possible de représenter ces données dans des systèmes d'information géographique (SIG) (outil informatique permettant de dresser des cartes cumulant diverses informations géographiques) et d'étudier des concordances lexicales entre des aires linguistiques et culturelles différentes.

Daniel Le Bris travaille actuellement dans ce domaine de la géolinguistique. Par une analyse motivationnelle du lexique comparé entre zones géographiques, il met en évidence des concordances aréales sur les franges atlantiques de l'Europe et des échanges mutuels entre lan-

9. Françoise Hammer, Der bretonische Dialekt von Ploubarnel (Phonologie, Morphophonologie, Morphologie und syntaktische Gruppen), thèse de doctorat, Université de Kiel, 1969.

10. Elmar TERnES, Grammaire structurale du breton de l'île de Groix, thèse de doctorat, Université de Heidelberg, 1970.

11. Loic Cheveau, Approche phonologique, morphologique et syntaxique du breton du Grand Lorient ( bas-vannetais), thèse de doctorat, Rennes, Université Rennes 2, 2007.

12. Maxime-Morvan CRAHÉ, Le breton de Languidic : étude phonétique, morphologique et syntaxique d'un sous-dialecte du breton vannetais, thèse de doctorat, Rennes, Université Rennes 2, 2013. 
gues celtiques, romanes et germaniques ${ }^{13}$. Les données locales collectées auprès de l'informateur de Plouhinec sont une modeste mais précieuse contribution à cette recherche de vaste envergure.

\section{Bibliographie}

Campbell A.-C. et Nicholls J., Guide de la faune et de la flore littorales des mers d'Europe, Neuchâtel, Delachaux et Niestlé, 1979.

CHeVeau Loic, Approche phonologique, morphologique et syntaxique du breton $d u$ Grand Lorient (bas-vannetais), thèse de doctorat, Université de Rennes 2, 2007.

CRAHÉ Maxime-Morvan, Le breton de Languidic : étude phonétique, morphologique et syntaxique d'un sous-dialecte du breton vannetais, thèse de doctorat, Rennes, Université Rennes 2, 2013.

FAVereau Francis, Grammaire du breton contemporain, Morlaix, Skol Vreizh, 1997.

GuilLEVIC A. et Le Goff P., Grammaire bretonne du dialecte de Vannes, Vannes, Lafolye et de Malarzelle, 1931.

HAMmer Françoise, Der bretonische Dialekt von Ploubarnel (Phonologie, Morphophonologie, Morphologie und syntaktische Gruppen), thèse de doctorat, Université de Kiel, 1969.

Herrieu Meriadeg, Le breton parlé, Vannes, Bleun Brug Bro Gwened, 1979.

LE BAYON Joseph, Grammaire bretonne du dialecte de Vannes, Vannes, Lafolye, 1896.

LE BERRE Alain, Ichtbyonymie bretonne, 3 volumes, Brest, CRBC, 1973.

LE BERRE Alain, Ichthyonymie bretonne, Atlas linguistique de la faune marine de Bretagne, Brest, CRBC et IUEM, 2008.

LE BRIS Daniel (dir.), Aires linguistiques / aires culturelles. Études de concordances en Europe occidentale: zones Manche et Atlantique, Brest, CRBC, 2012.

LE BRIS Daniel, "Concordances linguistiques celto-ibériques : les noms de "requin peau-bleue", prionace glauca, en Péninsule armoricaine et de "requin-taupe", lamna nasus, en péninsule ibérique », Estudis Romanics [Institut d'Estudis Catalans], vol. 35, 2013, p. 283-305.

13. Daniel LE BRIS (dir.), Aires linguistiques / aires culturelles. Études de concordances en Europe occidentale: zones Manche et Atlantique, Brest, CRBC, 2012 ; «Concordances linguistiques celto-ibériques : les noms de "requin peau-bleue", prionace glauca, en Péninsule armoricaine et de "requin-taupe", lamna nasus, en Péninsule ibérique», Estudis Romanics [Institut d'Estudis Catalans], vol. 35, 2013, p. 283-305. 
LE DÛ Jean, Nouvel Atlas Linguistique de Basse-Bretagne, 2 volumes, Brest, CRBC, 2001.

Le Roux Pierre, Atlas linguistique de la Basse-Bretagne, Rennes, Plihon et Hommay, E. Champion, 1924.

TERNES Elmar, Grammaire structurale du breton de l'Ile de Groix, thèse de doctorat, Université de Heidelberg, 1970. 